\title{
PERSPECTIVES
}

OPINION

\section{Vaccination against polio should not be stopped}

\section{Konstantin Chumakov, Ellie Ehrenfeld, Eckard Wimmer and Vadim I. Agol}

Abstract | The striking 50-year-long decline in the incidence of poliomyelitis has stalled in the past 7 years, which has led to calls for an urgent re-assessment of eradication and post-eradication campaign strategies. The current plan of eliminating the circulation of wild poliovirus so that further immunization will be unnecessary does not take into account recent scientific data and political realities that limit the likelihood that this strategy can sustain prevention of the disease. It is crucially important that high levels of population immunity are maintained against polio in the foreseeable future.

The earliest written documentation of poliomyelitis was provided by descriptions of the disease in the eighteenth and nineteenth centuries in Europe and North America (BOX 1). Epidemic outbreaks occurred on both continents in the late nineteenth century, which generated seasonal summertime fear until the 1950s. US President F. D. Roosevelt had been afflicted with poliomyelitis as a young man, which further encouraged an initiative to develop a vaccine for this disease. In 1955, an inactivated polio vaccine (IPV), developed by Jonas Salk, was licensed. Several years later, live, oral polio vaccine (OPV), developed by Albert Sabin, was introduced. Poliovirus occurs as three serotypes, but both vaccines are trivalent and provide excellent protection against poliomyelitis. Although a few countries have used IPV continuously, Sabin's OPV vaccine has been used to immunize individuals in most of the world owing to the ease of oral administration, the comparatively high level of intestinal immunity that it provides, its ability to spread to contacts of vaccine recipients and its lower cost.

The success of the polio vaccines in reducing the incidence of poliomyelitis inspired the World Health Assembly to launch a Global Polio Eradication Initiative (GPEI) in 1988. The initial plan for the GPEI was to eliminate polio worldwide by the year 2000 by implementing large-scale immunization campaigns with OPV. Massive national campaigns were to be conducted on numerous specified immunization days to achieve maximum coverage of all the children in the world. Although it was already recognized that the OPV strains occasionally reverted to neurovirulence, causing vaccine-associated paralytic poliomyelitis (VAPP) disease in vaccine recipients and their contacts, it was generally thought that these vaccine-derived viruses did not circulate widely. Therefore, if wild viruses were replaced by OPV-derived strains, it was reasoned that the extremely low frequency of disease that is caused by vaccination could, subsequently, be eliminated by the global cessation of immunization. Unfortunately, the past 18 years have revealed many facts that raise major concerns about this strategy of eradication of wild polioviruses, as well as the potential consequences of the cessation of vaccination ${ }^{1,2}$.

The GPEI has undergone significant evolution in response to emerging challenges over the course of its lifetime. Most recently, supplementary immunization with monovalent strains of OPV (type 1 or type 3 ) has been introduced in some regions where the virus has been most difficult to control or where outbreaks of a single serotype have occurred. Monovalent OPV is more potent in inducing an immune response than the trivalent vaccine, and it is hoped that it might tip the balance in the struggle to stop transmission. However, the ultimate objectives, definitions of success and strategies for the future of the programme remain essentially unchanged. Recent events on the global scene make it timely not only to reassess the tactics of stopping wild-poliovirus circulation, but also to chart policies beyond eradication. Here, we review the scientific basis that underlies these concerns and present our opinions: the failure to detect circulating wild poliovirus does not guarantee the elimination of polio infection; the current use of OPV is associated with a significant risk of polio epidemics that are caused by OPV-derivatives and, therefore, a gradual global switch to IPV should be encouraged; and, even if wildpoliovirus circulation is halted, leaving the majority of the world population susceptible to poliomyelitis will probably lead to new outbreaks of unpredictable severity. As a result, protection against poliovirus infection must continue into the foreseeable future, preferably as part of a routine paediatric vaccination schedule. We suggest that the goal of the polio campaign should be redefined to entail the long-term protection of the world population from poliomyelitis, rather than only the elimination of the detectable circulation of wild poliovirus.

\section{Current status of eradication}

Before the launch of the GPEI, two strategies were considered for the elimination of poliomyelitis. The first was based on creating and maintaining a level of global immunity that would prevent and, therefore, control the paralytic disease. The second relied on eradicating the transmission of wild polioviruses so that, it was argued, there would eventually be no need for vaccination. The choice was made in favour of eradication. The prospects of redirecting public health resources to other priorities following the eradication of poliovirus was a major factor that motivated governments and private institutions to commit to the poliovirus-eradication campaign. Huge logistic and financial resources were directed towards developing the infrastructure to achieve high vaccine coverage in endemic regions, sophisticated surveillance to trace virus transmission and mop-up operations to exterminate the remaining wild polioviruses. 


\section{Box $1 \mid$ History and pathogenesis of poliomyelitis}

Poliomyelitis (from the Greek polios (grey) and myelos (marrow); also known as infantile paralysis) was first recognized as a distinct human disease by Jacob Heine in 1840. It affects motor neurons of the anterior horns in the grey matter of the spinal cord. A major causative agent of poliomyelitis is poliovirus - an RNA-containing virus that belongs to the Enterovirus genus of the Picornaviridae family - but other infectious agents, including some other enteroviruses, can also cause the disease. In most cases, the infection of non-immune individuals with poliovirus causes no, or mild and reversible, symptoms. However, in approximately $1 \%$ of cases (or less, depending on the virus serotype), irreversible paralytic disease develops owing to the death of muscle-innervating neurons. Without nerve stimulation, the muscles become weak and undergo atrophy, eventually resulting in so-called acute flaccid paralysis. Sometimes, infection spreads into the brain stem (bulbar or bulbospinal disease), which can result in paralysis of the diaphragm and a severe impairment of breathing. A respirator is often required to sustain life (see the photograph, which depicts patients with poliomyelitis in a hospital respiratory ward in Los Angeles, United States, in 1952). The disease is usually more severe in adults than in children and can be fatal. A young man with a withered leg and foot, which is characteristic of patients with poliomyelitis, was depicted on an Egyptian stele made between 1403 and 1365 BCE (before the Common Era). It is considered likely that poliovirus was circulating at that time, approximately 3,400 years ago, although alternative causes cannot be ruled out. Photograph courtesy of the Centers for Disease Control and Prevention, Georgia, USA.

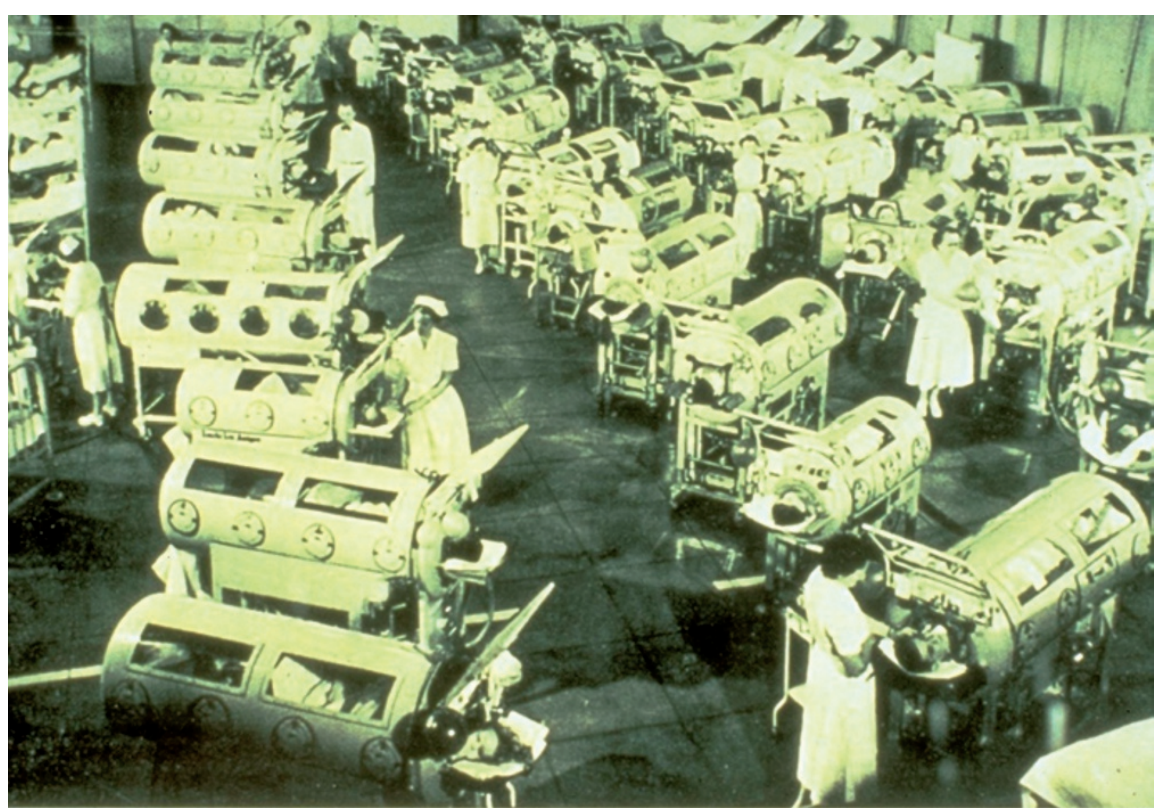

This multi-national effort was coordinated by national governments, the World Health Organization (WHO), Rotary International, the United States Centers for Disease Control, UNICEF (The United Nations Children's Fund) and private donors.

The campaign reduced poliomyelitis rates from an estimated 350,000 cases worldwide in 1988 to only 2,971 confirmed cases in 2000 , and completely eliminated the disease that is caused by wild-serotype- 2 poliovirus ${ }^{3}$. The 7 years that followed, however, were a mixture of successes and unanticipated setbacks and, despite a continuing reduction in the number of countries where circulation has never stopped (so-called polio-endemic countries), the global polio incidence remains at around 2,000 cases per year (FIG. 1). Officially, only four polioendemic countries remain: India, Pakistan,
Afghanistan and Nigeria. The failure to eliminate wild poliovirus in these remaining countries has probably been due to political and/or public health problems (BOX 2), as well as the inherent biological characteristics of the virus (discussed below). The reintroduction of wild poliovirus from these endemic regions into areas that were previously considered to be polio-free, followed by international secondary spread, has caused disease outbreaks in at least 25 countries in the past few years.

Challenges to the eradication programme Covert circulation of wild poliovirus. In 2004, wild polioviruses were identified that seemingly came 'out of nowhere'. These viruses, which are called orphan polioviruses, presented with genotypes that were neither related to nor derived from any known con- temporary viruses. Serotype-1 orphans were isolated in Sudan, and serotype-3 orphans were identified in Sudan and Chad. The closest relatives of these orphan polioviruses had been isolated from Central Africa 4-10 years earlier. Regardless of whether orphans reflect some failure of surveillance, isolation, identification or reporting, or whether they have emerged after long-term circulation without causing overt cases of polio, we should acknowledge that the absence of circulating wild polioviruses from official reports does not necessarily mean that they are absent from our planet. Thus, the number of countries in which endemic wild polioviruses are detected might be greater than four.

Circulation of vaccine-derived polioviruses. Although the OPV is a highly efficacious vaccine that is safe when it is used in a population with pre-existing anti-polio immunity, it is genetically unstable. OPV descendents rapidly acquire neurovirulence and transmissibility that closely resemble the phenotypes of wild polioviruses (FIG. 2). Ironically, this genetic instability was used by Sabin during the isolation of the polio vaccines $^{4}$, based on the understanding that each virus isolate contains a mixture of particles that express a spectrum of pathogenicity. As we now know, viral RNA-dependent RNA polymerases manifest high infidelity: each newly synthesized molecule of poliovirus RNA contains, on average, one mutation. Even clonal populations of poliovirus are highly heterogeneous 'swarms' of genotypes and phenotypes (reviewed in REF. 5). Sabin selected vaccine strains by adapting them to non-human cells, which resulted in attenuated neurovirulence in humans. During the subsequent passage in humans, however, the attenuating mutations are eliminated by natural selection. This leads to the restoration of the wild-type phenotype, for example, more robust replication, spreading and neurovirulence ${ }^{5,6}$. Even the OPV derivatives isolated from the faeces of a healthy vaccine recipient can exhibit a significant increase in virulence ${ }^{7}$. The rapid selection of viruses with improved fitness is not surprising as the genomes of the vaccine strains contain few major attenuating mutations (six in Sabin 1, two in Sabin 2 and three in Sabin 3) ${ }^{8,9}$.

In humans, the reversion to neurovirulence by OPV strains causes VAPP at a rate of approximately 1 in 500,000 first-OPV doses ${ }^{10,11}$. Although this was an acceptable level in the early stages of polio control and eradication, it is now estimated that 500 VAPP cases occur annually. Surprisingly, outbreaks of epidemic 


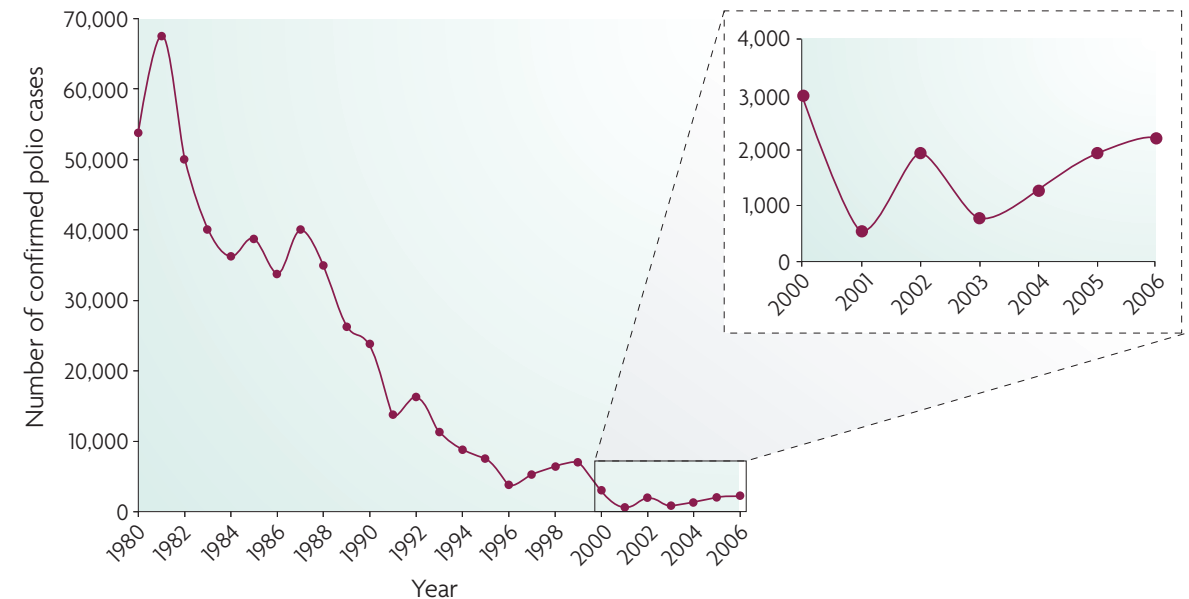

Figure 1 | Worldwide annual incidence of poliomyelitis. Data are taken from World Health Organization reports published in Morbidity and Mortality Weekly. The inset shows the data from the past 7 years on an expanded scale. poliomyelitis caused by vaccine-derived strains were discovered only recently ${ }^{12}$. The original eradication strategy was based on the misconception that although revertant polioviruses possess increased neurovirulence, their transmissibility remains low and they quickly disappear from populations. This hypothesis was disproved in 2000 when it was shown that numerous cases of poliomyelitis in Hispaniola had been caused by circulating vaccine-derived poliovirus (cVDPV) type 1 (REF. 12). Retrospective analysis showed that an earlier outbreak by cVDPVs occurred from 1988 to 1993 in Egypt and was caused by a virus that was less than $10 \%$ different from Sabin-2 virus. Circumstantial evidence sugpoliovirus that was circulating in Africa and Asia in the 1980s (assumed to be the wild type) was derived from Sabin-1 virus ${ }^{13}$.

The outbreaks caused by cVDPV that have occurred since 2000 (TABLE 1) have been relatively limited compared with previous years, generally paralyzing only a few individuals each, although 30 and 46 victims were confirmed during the Egyptian and Indonesian outbreaks, respectively ${ }^{14}$ (see Note added in proof). As only 1 in several-hundred people who are infected with wild poliovirus develops paralysis ${ }^{15}$, the number of infected individuals who were associated with these outbreaks was higher by at least 100 -fold. The limited spread of cVDPVs was probably due to the herd immunity that existed in the affected populations, although individual isolates of VDPV probably manifest varying degrees of virulence and transmissibility. Wild polioviruses also exhibit a wide range of virulence and transmissibility depending on their genetic properties and the immune status of their target populations. Indeed, in gests that the so-called genotype $\mathrm{T}$ of type-1 the pre-industrial era, when anti-polio immunity was almost universal, wild polioviruses caused only limited outbreaks. If allowed to continue to circulate, however, cVDPVs will probably evolve to become indistinguishable from wild polioviruses.

\section{Additional reservoirs of poliovirus. In} addition to the epidemic causing cVDPVs, there are other sources of pathogenic OPV derivatives. Vaccine recipients that have inborn immunodeficiency may excrete virus, which has been called iVDPV, for several years. The excretion of iVDPV has been described in at least 30 cases $^{14}$ and it can last for up to 20 years ${ }^{14,16}$. However, a search in the United States, Mexico, Brazil and the United Kingdom identified only a few cases of chronic polio carriers ${ }^{17}$, and the possibility that there are undiscovered polio excretors in the global human population of more than 6 billion people is probably a low, but undefined, risk. The evolution of the iVDPVs excreted by these patients appears to follow the same pattern as cVDPV, resulting in the early acquisition of increased pathogenicity ${ }^{18-20}$. Rare instances of the long-term excretion of OPV derivatives by healthy individuals who have no apparent immunopathology have also been documented ${ }^{21,22}$.

Subpopulations that have low or nonexistent immunity have been detected in some countries that have adequate levels of immunization. Such 'islands' are formed either owing to religious beliefs (such as Amish communities in the United States) or socio-economic conditions (for example, the Roma in Romania), and they might create reservoirs of highly diverged VDPV that can produce paralytic polio $0^{23,24}$.

Just as there are orphan wild polioviruses, there are also orphan VDPVs (also called ambiguous or aVDPV $\left.{ }^{14}\right)$. Highly divergent aVDPVs have been isolated from sporadic cases of paralytic polio and healthy children in populations considered to be adequately immunized ${ }^{25,26}$. Moreover, aVDPVs are occasionally isolated from sewage in regions that have no reported cases of poliomyelitis ${ }^{27-30}$.

The covert circulation of orphan wild polioviruses and the widespread occurrence of VDPVs that have properties approaching those of wild polioviruses seriously challenge the validity of the certification of regions as being 'free from wild polioviruses'. Certification is based on a failure to isolate wild poliovirus over a 3-year period. Considering the intrinsic properties of poliovirus and the lack of an absolutely reliable surveillance network, this policy is based more on faith than on fact, and it is, in our view, potentially dangerous. In most of the regions that are certified as polio-free, various OPV-derived poliovirus populations with wild-like properties are still circulating. The evidence suggests that the phenotypes

\section{Box 2 Obstacles to eradication in endemic countries}

A major cause of the continued transmission of wild polioviruses in the four remaining polioendemic regions is the failure to achieve sufficient vaccine coverage. In 2003, Nigeria deliberately stopped immunization campaigns owing to organized resistance by individuals who believed that the vaccine was contaminated with HIV-1 or anti-fertility drugs. Virus outbreaks subsequently occurred, which rapidly spread to neighbouring countries that had been polio-free, and the virus eventually found its way, through travellers, to distant locations such as Indonesia and Botswana. Although the misconception about contamination has officially been corrected, there is still a high percentage of children who receive no vaccine in some regions. In areas where war and armed conflict interfere with the maintenance and function of public health infrastructure, such as the Pakistan-Afghanistan border, health-care workers cannot safely deliver vaccine, despite efforts to cease violence on specified 'days of tranquillity'. Perhaps the most complex situation exists in the districts of Uttar Pradesh and Bihar in northern India. Although insufficient vaccine coverage may be part of the problem, there are documented cases of paralytic poliomyelitis in children who have received more than ten doses of vaccine. It is likely that the extreme poverty, overcrowding and poor sanitation in this region, which result in children being chronically infected with other enteric agents, interfere with vaccine efficacy and cause vaccine failure. 


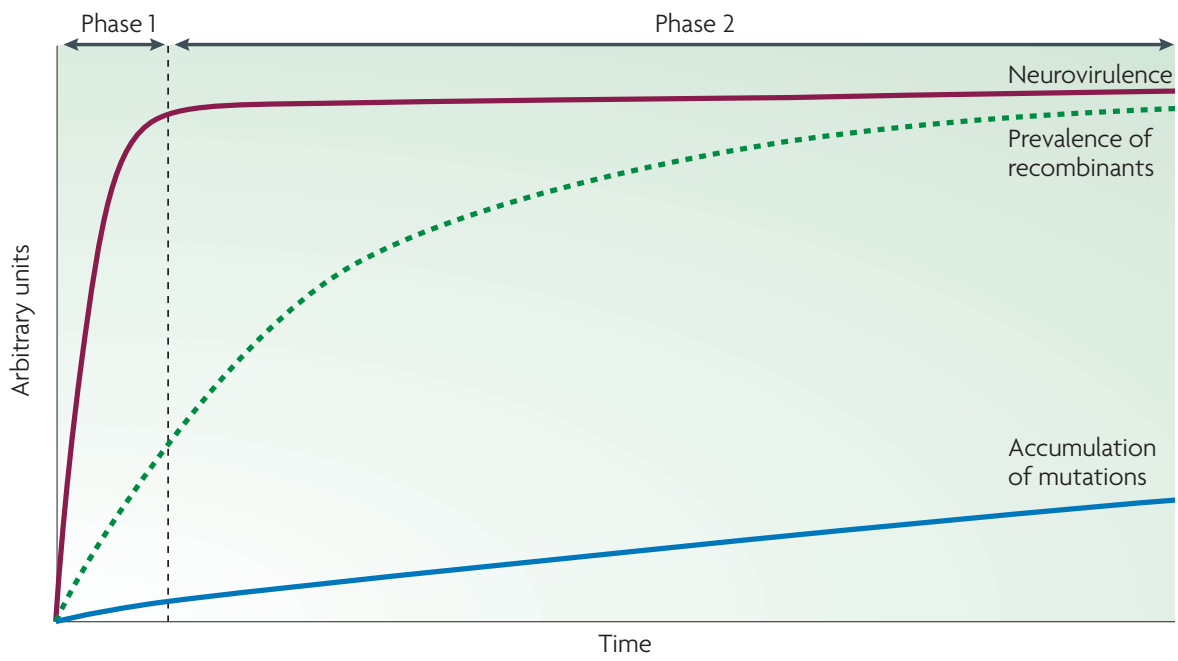

Figure 2 | A schematic representation of the time course of phenotypic and genetic changes in live, oral polio vaccine (OPV) derivatives. Although the pathways that lead to increased pathogenicity and spread are not known for any specific circulating vaccine-derived poliovirus (cVDPV) and might vary among viral lineages, some observations suggest that OPV descendants evolve in two phases $^{6,58}$. During the early phase (weeks after vaccination), attenuating mutations are eliminated, which leads to an increase in general fitness and neurovirulence that is similar to wild-type poliovirus. The more attenuating the mutations are, the more rapidly they are eliminated. During the second phase, no dramatic changes in neurovirulence occur, although mutations are accumulating nearly linearly in time ( $1 \%$ of nucleotides per year). These mutations are mainly phenotypically neutral as they are predominantly silent (codon synonymous). Among OPV derivatives, and especially cVDPVs, recombinants between circulating vaccine polioviruses and coxsackie $A$ viruses belonging to the human enterovirus $C$ species also accumulate with time and eventually become predominant ${ }^{8,59}$. Recombination between polio and non-polio enteroviruses can be reproduced in the laboratory ${ }^{60}$. The effect of these recombination events on VDPV pathogenicity and/or transmissibility is unknown.

of diverged VDPVs match (or will match) those of wild polioviruses and, consequently, VDPVs should be considered to be no different from wild polioviruses. New cases of cVDPVs have been discovered almost every year and thus it is, and will continue to be, difficult to accept certification of global polio eradication while OPV continues to be used.

Besides the continuous circulation of virus, there are other potential sources that could re-start the global polio epidemic should immunization programmes cease. These include: specimens collected from patients in clinical laboratories, which are related or unrelated to poliomyelitis; bioresearch; manufacturing establishments of vaccine; and, possibly, natural repositories of poliovirus-containing materials, such as permafrost ${ }^{31}$. Although considerable energy has been expended on the production of inventories of the existing stocks of poliovirus, there is no guarantee that all polioviruses can ever be destroyed or contained. Even if they could, the ease with which the poliovirus genome can be synthesized chemically ${ }^{32}$ could make such efforts useless. Extraordinary progress in synthetic biology $y^{33-35}$ means that virulent polioviruses will always remain within easy reach of those wanting to create terror and chaos. Therefore, although the containment of poliovirus is an important and worthy pursuit, we must not base our policies on the assumption that we can succeed in doing so.

Pathogenicity in non-immune populations In view of the uncertainties about the covert persistence of wild polioviruses and VDPVs, the safest public health policy is to leave no human population unprotected against poliovirus. It has been estimated that there are more people susceptible to poliovirus now than at any other time in history ${ }^{36}$. This situation will worsen if vaccination is stopped soon after the world is certified as being 'free from wild polioviruses' (REFS 37,38). Recent outbreaks caused by cVDPV, such as those discussed above, clearly demonstrate what can happen when the level of herd-anti-polio immunity is inadequate. Even more serious consequences could emerge in populations that completely lack immunity to polio.

An experiment in the Mogilev region (Byelorussian Republic of the former Soviet Union) in the 1960s has allowed us to evaluate the spread of poliovirus following the termination of OPV vaccination ${ }^{39}$. The immunization of children in the Mogilev region was intentionally interrupted between 1963 and 1966, except for a single cohort of 40 children who received a single dose of OPV in 1965. Unvaccinated children born after 1964 (who would not carry maternal antibodies) were then tested for polio antibodies and poliovirus. Nearly $40 \%$ of the children tested had antibodies to polio-type 2 , and approximately $15 \%$ had antibodies to 1 of the other 2 polio serotypes. Type- 2 polioviruses were isolated from approximately $10 \%$ of the children, and full-genome sequencing suggested that the polioviruses had not only been introduced into the community during the limited OPV vaccination, but also before and after it. Thus, in an immunologically naive population, polioviruses spread rampantly.

The Mogilev experiment raises concerns about proposals to eliminate Sabin-2 virus from the OPV vaccine ${ }^{40}$. Although no wild polioviruses of this serotype have been isolated since 1999 (REF. 8), highly neurovirulent type-2 polioviruses that exist in the form of cVDPV are likely to spread if non-immune children are exposed.

The spread of poliovirus in 1949 through the Chesterfield Inlet region of the North West Territories, Canada, probably also demonstrates the drastic consequences of immunological naivety to poliovirus.

A community of 275 people who lived in the Chesterfield region had been isolated from contact with the outside world for many decades, and no cases of poliomyelitis had been recorded during this time. When the poliovirus was introduced into the community by a traveller, 57 paralytic cases (20\% attack rate) and 14 deaths (25\% mortality) followed, and an unusually high proportion of adults were affected ${ }^{41-43}$. Although genetic (or other) factors might have influenced this extraordinary disease severity, the correlation with the absence of any immunity to poliovirus is striking. Similar outbreaks in 'virgin soil' communities also occurred in the Nicobar Islands, India, and the island of Saint Helena in 1947 (REF. 44).

\section{Combining control and eradication}

An infectious disease can be prevented either by the complete extermination of the pathogen or by creating and maintaining universal immunity to it. Extermination can only be viewed as a permanent solution if there is no possibility for the agent to resume circulation. The shortcoming of control is that it is an open-ended solution; however, unlike eradication it does not create massive vulnerability to the re-introduction of poliovirus. Ideally, disease prevention should include both strategies. 
The official polio-eradication strategy relies only on stopping poliovirus transmission and does not include long-term control, which is perhaps its biggest flaw. OPVs have led to a remarkable reduction in the incidence of poliomyelitis and the initial reason to propose stopping their use was financial. Most industrialized countries have already shifted from the eradication mode to the control mode by switching from the use of OPV to IPV. WHO has remained neutral regarding this transition, and has no plans to promote a similar switch in low-income countries. This is equivalent to intentionally leaving populations in resource-limited countries unprotected, and is unacceptable for ethical and political reasons and because it could jeopardize international public health and re-ignite a new global epidemic of poliomyelitis. A clear pro-active international plan for polio control must be a part of the eradication campaign, regardless of whether wild-poliovirus transmission is successfully eliminated.

A collection of recently published papers attempted to quantify the risks and estimate the costs that are associated with the different eradication and control scenarios ${ }^{45-47}$. The risk analysis involved the mathematical modelling of existing data based on certain assumptions. However, important parameters, such as the transmission and pathogenicity of polioviruses in a naive population, are unknown. On the one hand, the relationship between population immunity and the severity of disease during epidemics was not considered. On the other hand, the dangers of cVDPVs might have been over-estimated, as these only become a major threat if immunization is stopped. Such modelling is an elegant and useful research tool, but should not be used in isolation for developing major public health policies. The cost analyses of eradication and control scenarios are based on the premise that polio immunization will not be necessary once wild-poliovirus eradication is complete ${ }^{48}$. This represents a false assumption that there is a choice between eradication and control. In fact, eradication without subsequent control makes no sense and is not sustainable. So, although the current anti-polio struggle that is directed by WHO should continue unabated, the goal should be redefined from eliminating the last case of paralytic poliomyelitis caused by a wild poliovirus to achieving and maintaining the widest-possible protection against polio. That is, the programme should continue with vaccination regardless of whether polio has been eradicated.

\section{Strategies for control}

A plan that involves control must include two essential components: routine immunization of children and active surveillance, both virological and immunological. Each component presents substantial challenges because of competition with other public health priorities for resources, and the com placency that develops as the perception of urgency fades. Therefore, it is highly desirable that these activities be combined with other ongoing public health programmes and become a part of a wider campaign to control other vaccine-preventable diseases.

The ideal vaccine for a future routine immunization programme would be one that neither causes adverse reactions nor triggers the emergence of VDPVs, but that stimulates sufficient immunity to prevent the transmission of poliovirus. Several reports have described modified Sabin strains that are predicted to have increased genetic stability ${ }^{49-52}$. However, the enormous size of the clinical trial that is needed to prove the superiority of such strains over conventional Sabin strains in humans is daunting. Moreover, attenuating mutations outside the capsid region, no matter how stable, can be readily eliminated by recombination. Although a breakthrough in the invention of a new live polio vaccine cannot be ruled out, future immunization programmes would probably use inactivated vaccines, once financial and logistical issues are resolved.

The major disadvantage of IPV is its inadequate ability to induce intestinal immunity. Here lies a challenge to the research community: they should continue their efforts to improve IPV and produce a vaccine that combines the advantages of the two presentday vaccines. This could be done by using novel adjuvants and routes of administration, or by exploring other innovative approaches. Furthermore, the large-scale production of polyvalent vaccines that contain IPV combined with other protective antigens, which are already used in some industrialized countries, is required. Clinical studies of IPV and combination vaccines in countries with tropical climates have demonstrated that their immunogenicity, as measured by serum antibodies, is superior to that of OPV ${ }^{53-56}$. The use of combination vaccines throughout the world could provide an added public health benefit, as it could increase the costefficiency of the programme and prevent countries from dangerously stopping polio immunization for financial motives. The development of intradermal delivery devices could also help to solve the logistical problems of vaccine administration by injection, both for polio and for combination vaccine products. The additional costs that are associated with the introduction of IPV into multivalent vaccines for routine immunization would probably be mitigated in part by the increased manufacturing scale. This cost represents a necessary premium for insurance against the return of poliomyelitis, and may be insignificant compared with the potentially devastating consequences of being unprepared for this possibility.

An integral part of any control strategy is a plan for an emergency response to the possible re-introduction of polio into circulation. The solution is straightforward if the level of population immunity is high. Using OPV in such a circumstance would

\section{Table 1 | Recent outbreaks of paralytic polio caused by circulating vaccine-derived polioviruses}

\begin{tabular}{lllll|}
\hline Country & Serotype & Number of known cases & Recombination with HEV-C & Year \\
\hline Indonesia & 1 & 46 & Yes & 2005 \\
\hline Madagascar & 2,3 & 7 & Yes & 2002,2005 \\
\hline China & 1 & 2 & No & 2004 \\
\hline Cambodia & 3 & 3 & Yes & $2005-2006$ \\
\hline Philippines & 1 & 1 & Yes & 2001 \\
\hline Hispaniola & 1 & 21 & Yes & 2000 \\
\hline Egypt & 2 & 30 & Yes & $1988-1993$ \\
\hline
\end{tabular}

Data from Ref. 14. HEV-C, C-cluster human enteroviruses (specifically coxsackie A viruses belonging to the human enterovirus $\mathrm{C}$ species). 
be similar to the current strategy to stop transmission of the wild virus; however, the potential disease burden that would be imposed by re-seeding immunologically naive populations with live virus is unpredictable. The 1949 incident in Canada and the speed at which Sabin strains revert to virulence by point mutations and/or recombination and spread in populations with suboptimal herd immunity are already causes for concern. Other complications that are associated with stopping the use of OPV include the regulation and maintenance of OPV stockpiles in newly restricted OPV-production facilities. Were vaccination to continue, these extraordinary containment requirements would be unnecessary. Moreover, novel anti-polio drugs could be developed, and these could be of great benefit for post-exposure prophylaxis and outbreak control ${ }^{57}$.

\section{Concluding remarks}

The evidence summarized above strongly suggests that any strategy that renders human populations vulnerable to infection by poliovirus is associated with an unacceptable risk. Therefore, the goal of the polio-eradication programme should be to achieve the widestpossible vaccination coverage. We may have no other choice than to continue immunization against a non-existing disease to prevent ourselves from becoming hostages to the threat of the devastating return of poliomyelitis. The short-term choice between OPV and IPV should depend on local conditions. The combination of IPV with other routine immunizations against important paediatric infections will be optimal in the long term.

Continued research on poliovirus should be encouraged and should focus on the improvement of the existing IPV. These improvements would include cost reduction, bundled delivery with other vaccines for children and boosting its ability to induce local immunity. In addition, the development of fundamentally new vaccines, as well as efficacious anti-polio drugs, should be explored. Such efforts could radically change vaccination policy decisions and eventually lead to the true eradication of poliomyelitis.

\section{Note added in proof}

Morbidity and Mortality Weekly Report has described an ongoing outbreak of poliomyelitis in Nigeria that is caused by type- 2 $\mathrm{cVDPV}^{61}$. It appears that there are multiple independent lineages of virulent virus, derived from the type-2 strain in trivalent OPV, which have caused approximately
100 cases of poliomyelitis in a broad area of northern Nigeria during the past 2 years. This outbreak could be an indicator of the transmission and evolution of VDPV in a population that has inadequate immunization coverage.

Konstantin Chumakov is at the Center for Biologics Evaluation and Research, Food and Drug Administration, 1401 Rockville Pike, Rockville, Maryland 20852, USA.

Ellie Ehrenfeld is at the National Institute of Allergy and Infectious Diseases, National Institutes of Health, Bethesda, Maryland 20892, USA.

Eckard Wimmer is at the Department of Molecular Genetics and Microbiology, Stony Brook University, School of Medicine, Stony Brook, New York, New York 11794, USA.

Vadim I. Agol is at the M.P. Chumakov Institute of Poliomyelitis and Viral Encephalitides, Russian Academy of Medical Sciences, Moscow 142782, Russia.

Correspondence to E.E. e-mail: eehrenfeld@niaid.nih.gov doi:10.1038/nrmicro1796 Published online 22 October 2007

Arita, I., Nakane, M. \& Fenner, F. Public health. Is polio eradication realistic? Science 312, 852-854 (2006).

2. Minor, P. D. Polio eradication, cessation of vaccination and re-emergence of disease. Nature Rev. Microbiol. 2, 473-482 (2004).

3. Apparent global interruption of wild poliovirus type 2 transmission. MMWR 50, 222-224 (2001).

4. Sabin, A. \& Boulger, L. History of Sabin attenuated poliovirus oral live vaccine strains. J. Biol. Stand. 1, 115-118 (1973).

5. Agol, V. I. Molecular mechanisms of poliovirus variation and evolution. Curr. Top. Microbiol. Immunol. 299, 211-259 (2006)

6. Agol, V. I. Vaccine-derived polioviruses. Biologicals 34 , 103-108 (2006).

7. Minor, P. \& Almond, J. in Molecular Biology of Picornaviruses (eds Semler, B. \& Wimmer, E.) 381-390 (American Society of Microbiology, Washington DC, 2002)

8. Kew, O. M., Sutter, R. W., de Gourville, E. M., Dowdle, W. R. \& Pallansch, M. A. Vaccine-derived polioviruses and the endgame strategy for global polio eradication. Annu. Rev. Microbiol. 59 587-635 (2005).

9. Mueller, S., Wimmer, E. \& Cello, J. Poliovirus and poliomyelitis: a tale of guts, brains, and an accidental event. Virus Res. 111, 175-193 (2005)

10. Nkowane, B. M. et al. Vaccine-associated paralytic poliomyelitis. United States: 1973 through 1984. JAMA 257, 1335-1340 (1987).

11. Prevots, D. R., Sutter, R. W., Strebel, P. M., Weibel, R. E. \& Cochi, S. L. Completeness of reporting for paralytic poliomyelitis, United States, 1980 through 1991. Implications for estimating the risk of vaccineassociated disease. Arch. Pediatr. Adolesc. Med. 148, 479-485 (1994).

12. Kew, O. et al. Outbreak of poliomyelitis in Hispaniola associated with circulating type 1 vaccine-derived poliovirus. Science 296, 356-359 (2002).

13. Lipskaya, G. et al. Geographical genotypes (geotypes) of poliovirus case isolates from the former Soviet Union: relatedness to other known poliovirus genotypes. J. Gen. Virol. 76, 1687-1699 (1995)

14. Update on vaccine-derived polioviruses. MMWR 55 , 1093-1097 (2006)

15. Melnick, J. in Virology (ed. Fields, B.) 739-794 (Raven, New York, 1985)

16. MacLennan, C. et al. Failure to clear persistent vaccinederived neurovirulent poliovirus infection in an immunodeficient man. Lancet 363, 1509-1513 (2004).

17. Halsey, N. A. et al. Search for poliovirus carriers among people with primary immune deficiency diseases in the United States, Mexico, Brazil, and the United Kingdom. Bull. World Health Organ. 82, 3-8 (2004).

18. Gavrilin, G. V., Cherkasova, E. A., Lipskaya, G. Y., Kew, O. M. \& Agol, V. I. Evolution of circulating wild poliovirus and of vaccine-derived poliovirus in an immunodeficient patient: a unifying model. J. Virol. 74, 7381-7390 (2000).

19. Martin, J., Dunn, G., Hull, R., Patel, V. \& Minor, P. D. Evolution of the Sabin strain of type 3 poliovirus in an immunodeficient patient during the entire 637-day period of virus excretion. J. Virol. 74 3001-3010 (2000).

20. Yang, C. F. et al. Intratypic recombination among lineages of type 1 vaccine-derived poliovirus emerging during chronic infection of an immunodeficient patient. J. Virol. 79, 12623-12634 (2005).

21. Hovi, T., Lindholm, N., Savolainen, C., Stenvik, M. \& Burns, C. Evolution of wild-type 1 poliovirus in two healthy siblings excreting the virus over a period of 6 months. J. Gen. Virol. 85, 369-377 (2004).

22. Martin, J. et al. Long-term excretion of vaccinederived poliovirus by a healthy child. J. Virol. 78, 13839-13847 (2004).

23. Poliovirus infections in four unvaccinated children - Minnesota, August-October 2005. MMWR 54 1053-1055 (2005)

24. Combiescu, M. et al. Circulation of a type 1 recombinant vaccine-derived poliovirus strain in a limited area in Romania. Arch. Virol. 152, 727-738 (2007).

25. Cherkasova, E. et al. Microarray analysis of evolution of RNA viruses: evidence of circulation of virulent highly divergent vaccine-derived polioviruses. Proc. Natl Acad. Sci. USA 100, 9398-9403 (2003).

26. Cherkasova, E. A. et al. Long-term circulation of vaccine-derived poliovirus that causes paralytic disease. J. Virol. 76, 6791-6799 (2002)

27. Blomqvist, S. et al. Characterization of a highly evolved vaccine-derived poliovirus type 3 isolated from sewage in Estonia. J. Virol. 78, 4876-4883 (2004).

28. Cernakova, B. et al. Isolation of vaccine-derived polioviruses in the Slovak Republic. Eur. J. Clin. Microbiol. Infect. Dis. 24, 438-439 (2005).

29. Horie, H. et al. Isolation of vaccine-derived type 1 polioviruses displaying similar properties to virulent wild strain Mahoney from sewage in Japan. J. Med. Virol. 68, 445-451 (2002).

30. Shulman, L. M. et al. Molecular and antigenic characterization of a highly evolved derivative of the type 2 oral poliovaccine strain isolated from sewage in Israel. J. Clin. Microbiol. 38, 3729-3734 (2000).

31. Dowdle, W. et al. Containment of polioviruses after eradication and OPV cessation: characterizing risks to improve management. Risk Anal. 26, 1449-1469 (2006).

32. Cello, J., Paul, A. V. \& Wimmer, E. Chemical synthesis of poliovirus CDNA: generation of infectious virus in the absence of natural template. Science 297, 1016-1018 (2002).

33. Tian, J. et al. Accurate multiplex gene synthesis from programmable DNA microchips. Nature $\mathbf{4 3 2}$ 1050-1054 (2004).

34. Tumpey, T. M. et al. Characterization of the reconstructed 1918 Spanish influenza pandemic virus. Science 310, 77-80 (2005)

35. Wimmer, E. The test-tube synthesis of a chemical called poliovirus. The simple synthesis of a virus has far-reaching societal implications. EMBO Rep. 7 S3-9 (2006).

36. MacKenzie, D. Eradicating polio leaves people defenceless. New Sci. 2562, 8 (2006).

37. Aylward, R. B., Sutter, R. W. \& Heymann, D. L. Policy. OPV cessation - the final step to a "poliofree" world. Science 310, 625-626 (2005).

38. Heymann, D. L., Sutter, R. W. \& Aylward, R. B. $A$ vision of a world without polio: the OPV cessation strategy. Biologicals 34, 75-79 (2006).

39. Korotkova, E. A. et al. Retrospective analysis of a local cessation of vaccination against poliomyelitis: a possible scenario for the future. J. Virol. 77 12460-12465 (2003).

40. John, T. J. Two good reasons to drop type 2 virus from oral polio vaccine. Lancet 364, 1666 (2004).

41. Peart, A. F., Rhodes, A. J. et al. An outbreak of poliomyelitis in Canadian Eskimos in wintertime. Can. J. Public Health 40, 405-419 (1949).

42. Rhodes, A. J. \& Chapman, M. Some observations on interference between neurotropic viruses. Can. J. Res. 27, 341-348 (1949). 


\section{PERSPECTIVES}

43. Rutty, C. The Middle Class Plague: Canada and the Polio Years. Thesis, Univ. Torronto (2005).

44. Burnet, F. Viral and infectious diseases. Annu. Rev. Med. 2, 1-24 (1951)

45. Tebbens, R. J. et al. Risks of paralytic disease due to wild or vaccine-derived poliovirus after eradication. Risk Anal. 26, 1471-1505 (2006).

46. Thompson, K. M., Duintjer Tebbens, R. J. \& Pallansch, M. A. Evaluation of response scenarios to potential polio outbreaks using mathematical models. Risk Anal. 26, 1541-1556 (2006).

47. Thompson, K. M. et al. Development and consideration of global policies for managing the future risks of poliovirus outbreaks: insights and lessons learned through modeling. Risk Anal. 26, 1571-1580 (2006).

48. Thompson, K. M. \& Tebbens, R. J. Eradication versus control for poliomyelitis: an economic analysis. Lancet 369, 1363-1371 (2007).

49. Agol, V. I., Pilipenko, E. V. \& Slobodskaya, O. R. Modification of translational control elements as a new approach to design of attenuated picornavirus strains. J. Biotechnol. 44, 119-128 (1996).

50. Kohara, M., Abe, S., Yoshioka, I. \& Nomoto, A. Development of candidates for new type 2 and type 3 oral poliovirus vaccines. Dev. Biol. Stand. 78 . 141-148 (1993)

51. Macadam, A. J. et al. Rational design of genetically stable, live-attenuated poliovirus vaccines of all three serotypes: relevance to poliomyelitis eradication. J. Virol. 80, 8653-8663 (2006)

52. Toyoda, H., Yin, J., Mueller, S., Wimmer, E. \& Cello, J. Oncolytic treatment and cure of neuroblastoma by a novel attenuated poliovirus in a novel poliovirussusceptible animal model. Cancer Res. 67 , 2857-2864 (2007).

53. Randomized, placebo-controlled trial of inactivated poliovirus vaccine in Cuba. N. Engl. J Med. 356, 1536-1544 (2007).

54. Melnick, J. L. Combined use of live and killed vaccines to control poliomyelitis in tropical areas. Dev. Biol. Stand. 47, 265-273 (1981)

55. Oduntan, S. O. \& Familusi, J. B. An appraisal of polio immunization in Nigeria and other tropical African Countries. Niger. Med. J. 9, 645-649 (1979).

56. Salk, D. \& Salk, J. Vaccinology of poliomyelitis. Vaccine 2, 59-74 (1984)

57. Workshop Report, Committee on Development of a Polio Antiviral and Its Potential Role in Global Poliomyelitis Eradication (ed. Press, T. N. A.) (National Research Council of the National Academies, Washington DC, 2006).

58. Yakovenko, M. L. et al. Antigenic evolution of vaccine-derived polioviruses: changes in individual epitopes and relative stability of the overall immunological properties. J. Virol. 80, 2641-2653 (2006).

59. Arita, M. et al. A Sabin 3-derived poliovirus recombinant contained a sequence homologous with indigenous human enterovirus species $\mathrm{C}$ in the viral polymerase coding region. J. Virol. 79 12650-12657 (2005).

60. Jiang, P. et al. Evidence for emergence of diverse polioviruses from C-cluster coxsackie A viruses: implications for global poliovirus eradication. Proc. Natl Acad. Sci. USA 104, 9457-9462 (2007).

61. Update on vaccine-derived polioviruses - worldwide January 2006-August 2007. MMWR 56, 996-1001 (2007).

\section{Acknowledgements}

Experimental work in the authors' laboratories is supported in part by research grants from the National Institutes of Health (E.W.), the intramural research programmes of the National Institute of Allergy and Infectious Diseases, the National Institutes of Health (E.E.) and the Food and Drug Adminstration (K.C.), the Russian Fund for Basic Research and the Program for Support of Leading Scientific Schools (V.A.). The opinions that are expressed in this article are those of the individual authors and do not represent those of their institutional affiliates.

DATABASES

Entrez Genome: http://www.ncbi.nlm.nih.gov/entrez/query. fcgi?db=genome 